\title{
Malignant Peripheral Nerve Sheath Tumor of the Orbit: Case Report and Literature Review
}

\author{
Mehmet D. Aydin, M.D., ${ }^{1}$ Umran Yildirim, M.D., ${ }^{2}$ Cemal Gundogdu, M.D., ${ }^{2}$ \\ Osman Dursun, M.D., ${ }^{3}$ Hasan H. Uysal, M.D., ${ }^{3}$ and Mete Ozdikici, M.D. ${ }^{4}$
}

\begin{abstract}
A 68-year-old woman with progressive visual loss and exophthalmos in her right eye had been operated on for a mass in her right calf 3 years earlier. Imaging showed a huge mass invading the orbital structures and temporal pole. The presumptive diagnosis was a malignant orbital tumor. The tumor was resected totally and eroded tissues such as the lateral rectus muscle and dural compartments were repaired. The histological diagnosis was a malignant peripheral nerve sheath tumor (MPNST). The patient recovered uneventfully and was discharged 8 days after surgery. Two years later she died from a liver tumor. Few MPNSTs involving the orbit have been reported.
\end{abstract}

KEYWORDS: Malignant peripheral nerve sheath tumor, orbit

Malignant peripheral nerve sheath tumors (MPNSTs) are rare and usually arise from peripheral nerves or somatic soft tissues. ${ }^{1,2}$ They constitute $5 \%$ of all sarcomas, and 70\% are associated with neurofibromatosis type 1 (NF1), the most common familial cancer-predisposing syndrome in humans. ${ }^{3,4}$ The incidence of neurofibrosarcomas in NF1 has ranged between $2 \%$ and 29\%, accounting for about $50 \%$ of these tumors. ${ }^{1,3,4,10}$ MPNSTs can develop in any anatomical region, but the sciatic nerve is affected most often. ${ }^{5}$ MPNSTs involving the face are extremely rare. They include heterologous mesenchymal and epithelial elements with increased mitotic activity. ${ }^{6}$ The definitive diagnosis of MPNST is obtained by biopsy. Metastasis occurs in $39 \%$ of patients and $68 \%$ die from their tumor. ${ }^{3}$ Surgery is the most benefical treatment, but postoperative radio- and chemotherapy are part of adjunctive therapy. ${ }^{7}$ We removed an MPNST from our patient through a lateral orbitozygomatic

Skull Base, volume 14, number 2, 2004. Address for correspondence and reprint requests: Dr. Mehmet D. Aydin, Atatürk Üniversitesi Lojmanlari, 60 Blok, Kat 1, No. 3, Erzurum, Turkey. E-mail: nmda21@hotmail.com. Departments of ${ }^{1}$ Neurosurgery and ${ }^{2}$ Pathology, Medical Faculty, Ataturk University, Erzurum, Turkey; ${ }^{3}$ Department of Ophthalmology, Social Security Hospital, Erzurum, Turkey; ${ }^{4}$ Department of Radiology, Erzurum State Hospital, Erzurum, Turkey. Copyright (C) 2004 by Thieme Medical Publishers, Inc., 333 Seventh Avenue, New York, NY 10001, USA. Tel: +1(212) 584-4662. 1531-5010,p;2004,14,02,109,114,ftx,en;sbs00393x. 
approach. Problems with cosmetic, vision, and ocular movements resolved after surgery.

\section{CASE REPORT}

A 68-year-old woman suffered from gradually increased blurred vision and exophthalmos of the right eye for 6 months. Six months before this presentation, proptosis developed on her right side. Ten days before she was hospitalized, she was blind in her right eye and the exophthalmos had advanced. Upon physical examination, visual acuity in her right eye was $1 / 20$. In addition to amaurosis, the exophthalmic right eye showed limited upward gaze and no lateral gaze. Her palpebras exhibited prominent chemosis. Her cornea was intact (Fig. 1A). The round pupil was $6 \mathrm{~mm}$ across and not reactive to light. The disc of the right optic nerve was edematous.

Magnetic resonance imaging (MRI) of the orbits and brain with and without contrast enhancement in the axial plane showed a large soft-tissue mass at the lateral side of the optic nerve. The tumor invaded the lateral rectus muscle and right temporal pole (Fig. 1B). Other systemic observations of the patient were normal.
Two years earlier, she had undergone resection of a painful tumor in the right calf. Histologic analysis of the tumor had suggested a malignant neurofibroma. Macroscopically, the resection was considered radical.

On this occasion, the tumor was exposed through a right orbitozygomatic approach. The tumor was highly vascularized and had eroded the zygomatic and sphenoid bones, lateral orbital muscle, sphenoid wing, dura, and temporal pole. The lesion was removed totally with microsurgical technique, and the lateral rectus muscle was repaired using a flap of temporal muscle. Duroplasty was performed using temporal fascia to repair the dural defect of the temporal pole. Microscopically, malignant differentiation and infiltration into the lateral rectus muscle were observed (Fig. 2A). The histopathological diagnosis was an MPNST (Figs. 2B,C). Postoperatively, the patient recovered rapidly. At discharge, she had no dramatic cosmetic facial problems. Vision and movement of her right eye were normal and her neurological status was stable (Fig. 3A). Six months after surgery, computed tomography (CT) showed no residual mass in the orbit (Fig. 3B), and radiotherapy was not recommended. The patient died of progressive liver tumor 2 years later. An autopsy was not allowed.

A
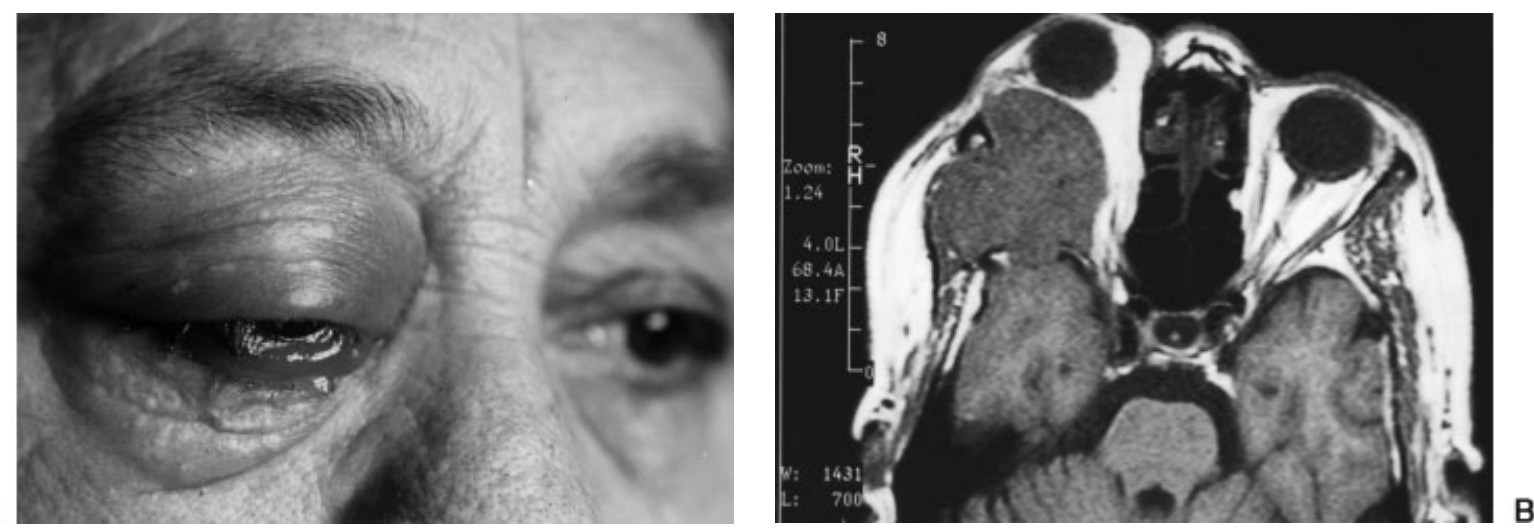

Figure 1 (A) Prominent exophthalmos, chemosis, and flashing in the palpebras in the patient's right eye. (B) T1-weighted $\mathrm{MRI}$ showing the lesion originating from the lateral part of the orbit. 
A
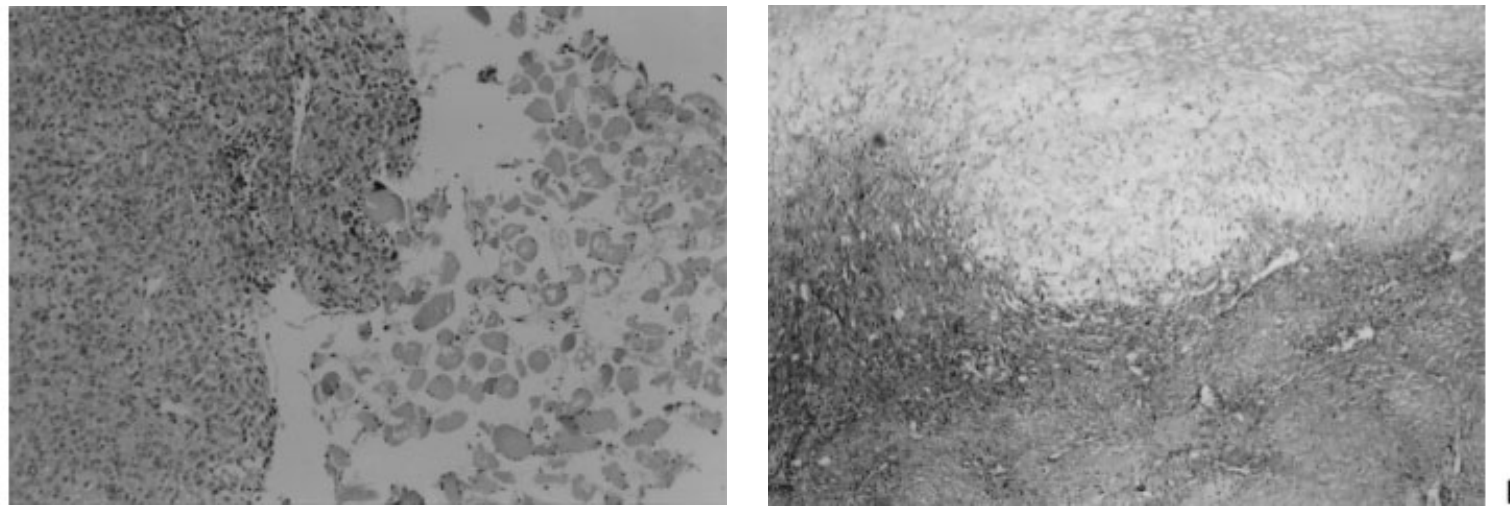

B

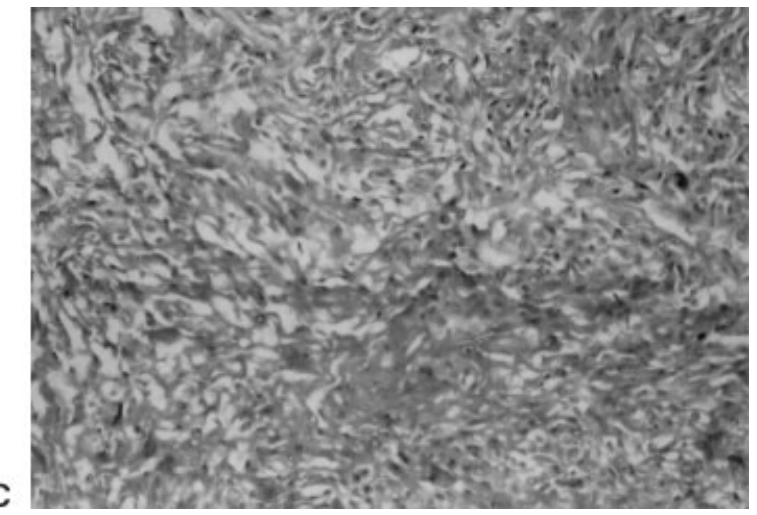

Figure 2 (A) Malignant tumoral cells and infiltrated tissue of the lateral rectus muscle (hematoxylin and eosin (H\&E), $\times 400)$. (B) Mixoid changes are visible $(H \& E, \times 100)$. (C) Rounded and ovoid cells include dense vascular and high mitotic activity $(H \& E, \times 100)$.

A
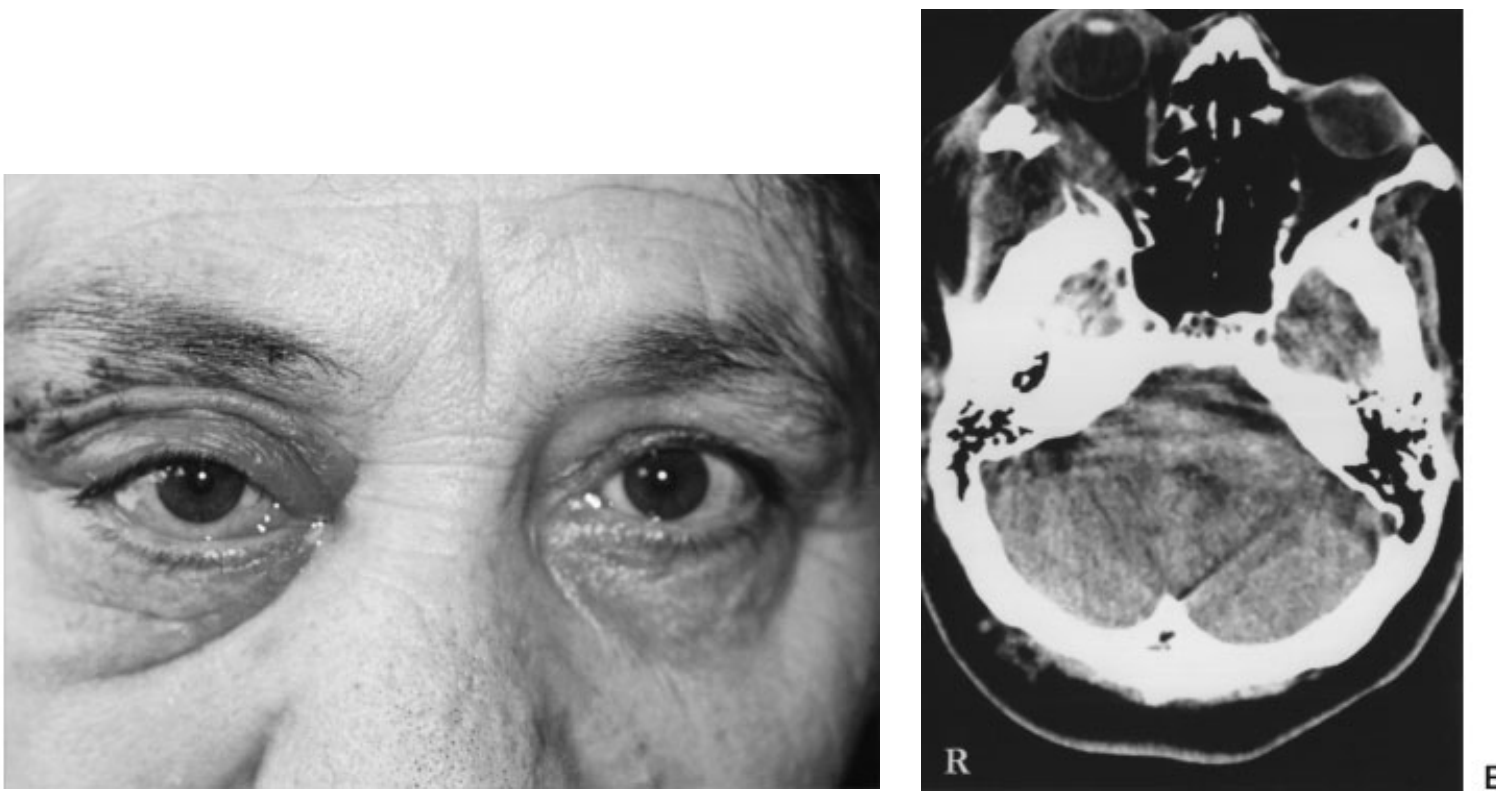

Figure 3 (A) Postoperatively, the patient had no obvious cosmetic problems and deviation of her eye resolved. (B) Postoperative computed tomography showed no residual mass in the orbit 6 months after surgery. The repaired lateral rectus muscle appears normal. 


\section{DISCUSSION}

Most MPNSTs are large, fleshy, often necrotic neoplasms that may involve proximal portions of major nerve trunks of the neck, extremities, retroperitoneum, mediastinum, and viscera. Less commonly, intradural nerves are involved. Although MPNSTs can develop in any anatomical region, the sciatic nerve is most often affected. ${ }^{5}$ Such tumors usually occur in large nerve trunks in patients with NF1 and are associated with a highly aggressive course of recurrence, metastases, and death in most patients. ${ }^{8}$ To our knowledge, few patients with a cranial or facial MPNST have been reported. ${ }^{1,2}$ Likewise, cranial nerves are rarely affected, although tumors of the trigeminal and acoustic nerves have been reported. $^{9}$

MPNSTs are fusiform to globular in shape and vary from white and firm to yellow and soft, depending on the absence or presence of necrosis. In most tumors, recognizable nerve roots enter or exit the tumors. Although the tumors usually appear well circumscribed, they are not truly encapsulated. On microscopic examination, they are often observed to invade soft tissue and nerve fascicles. ${ }^{11} \mathrm{In}$ our patient, tumor invaded the lateral rectus muscle of the eyeball. In $61 \%$ of the cases, ${ }^{5}$ the greatest dimension of the primary tumor is greater than $5 \mathrm{~cm}$.

The histologic features of MPNSTs are those of a highly cellular, spindle-cell neoplasm resembling a soft-tissue sarcoma, but with differentiation toward elements of the nerve sheath, Schwann cell, and perineural cell. ${ }^{12}$ Frequent mitoses and focal necrosis are typical. Cytologic features also vary and may include fibroblastic, schwannian, epitheloid, clear cell, or frankly anaplastic-pleomorphic cells. Rarely are heterologous mesenchymal or epithelial elements present. As in our case, MPNSTs can include heterologous mesenchymal and epithelial elements. Such atypical components show hypercellularity, an increased nuclear-to-cytoplasmic ratio, cytological atypia, and increased mitotic activity. ${ }^{6}$
Metastases occur in 39\% of patients. ${ }^{3,11}$ The most common metastatic sites are the lungs, followed in decreasing order of frequency by soft tissue, bone, liver, intra-abdominal cavity, adrenal glands, diaphragm, mediastinum, brain, ovaries, kidneys, and retroperitoneum. Sixty-eight percent of reported patients have died of tumor, and 32\% have either been lost to follow-up or died of unrelated causes at intervals ranging from 1 month to 23 years. ${ }^{4,11}$ The most important features adversely influencing prognosis are the presence of von Recklinghausen's disease, a tumor larger than $5 \mathrm{~cm}$, and extent of resection. ${ }^{11}$

Cranial CT shows a well-defined iso- or hypodense mass. Contrast-enhancement of these lesions shows persistent low-density foci corresponding to necrosis. The foci are suggestive of the malignant potential of the lesion and seem to indicate a poor prognosis. MRI shows a welldefined lesion with mixed components. Gadolinium-enhancement has occurred in all cases to variable degrees. ${ }^{13,14}$ In some cases, T2-weighted MRIs show small high-signal foci, suggesting central cystic necrosis. Cystic components may suggest either a benign schwannoma or the necrotic changes associated with a malignant schwannoma. Muscle invasion has been particularly well demonstrated on MRI and confirmed by surgery. ${ }^{13,14}$ In our patient, muscle invasion was detected at surgery and confirmed by histopathologic analyses (Fig. 3).

Biopsy is necessary to diagnose an MPNST definitively. The rhabdomyoblastic type is more often encountered in the head and neck. The differential diagnosis between benign schwannoma and neurosarcoma may be challenging: one must look for necrotic foci, the number of atypical mitoses, and an absence of differentiated cells. Tumors larger than $5 \mathrm{~cm}$, histological grades II and III, an association with neurofibromatosis, and regional or distant metastases suggest an ominous prognosis.

The treatment of choice is surgery, but postoperative radio- and chemotherapy are part of adjunctive therapy. ${ }^{7}$ Gross total resection of the 
tumor is the most important therapeutic goal. Wide excision or amputation (when extremities are involved) may be required in some cases. When radical tumor removal is not possible, excision combined with high-dose radiation therapy seems to be the best alternative treatment. ${ }^{11}$

\section{REFERENCES}

1. D'Agostino AN, Soule EH, Miller RH. Sarcomas of the peripheral nerves and somatic soft tissue associated with multiple neurofibromatosis (von Recklinghausen's disease). Cancer 1963;16:1015-1027

2. Grnja V, Allen WE, Osborn DJ, Kier EL. Sacral neurofibrosarcoma: an angiographic evaluation. Case report. J Neurosurg 1974;40:767-771

3. Wanebo J, MalikJ, Vanden Berg S, Wanebo H, Driesen N, Persing J. Malignant peripheral nerve sheath tumors. Cancer 1993;71:1247-1253

4. Wick M, Swanson P, Scheithauer B, Manivel J. Malignant peripheral nerve sheath tumor. Am J Clin Pathol 1987;87: 425-433

5. Kchouk M, Rabet AM, Ghedas K, et al. Extensive malignant schwannoma of the sciatic nerve. Contribution of imaging techniques [in French]. J Radiol 1993;74:641-644

6. Enzinger FM, Weiss SW. Soft Tissue Tumors. St Louis, MO: CV Mosby; 1983:625-654

7. Aguiar Vitacca S, Sarrazin D, Henry-Amar M, et al. Neurosarcoma associated with von Recklinhausen disease: apropos of 25 cases observed at the Gustave Roussy Institute from 1967 to 1990 [in French]. Bull Cancer 1992;79: 101-112

8. Robey SS, deMent SH, Eaton KK, Aoun H. Malignant epitheloid peripheral nerve sheath tumor arising in a benign schwannoma. Surg Neurol 1987;28:441-446

9. Han DH, Kim DG, Chi JG, Park SH, Jong HW, Kim YG. Malignant triton tumor of the acoustic nerve: case report. J Neurosurg 1992;76:874-877

10. Preston FW, Walsh WS, Clarke TH. Cutaneous neurofibromatosis (von Recklinghausen's disease): clinical manifestation and incidence of sarcoma in sixty-one male patients. AMA Arch Surg 1952;64:813-827

11. Barbara SD, Bernd WS, David GP, Herbert MR, Duane MI. Malignant peripheral nerve sheath tumors. Cancer 1986;57:2006-2021

12. Chitale AR, Dickersin GR. Electron microscopy in the diagnosis of malignant schwannomas. A report of six cases. Cancer 1983;51:1448-1461

13. Colmero C, Rives T, Patron M, Sierra I, Gamallo C. Maxillofacial malignant peripheral nerve sheath tumours. J Craniomaxillofac Surg 1991;19:40-46

14. Auclair P, Langloss J, Weiss S, Corio R. Sarcomas and sarcomatoid neoplasms of the major salivary gland regions.
A clinicopathologic and immunohistochemical study of 67 cases and review of the literature. Cancer 1986;58:13051315

\section{Commentary}

The authors have described an unusual presentation of an orbital and optic sheath tumor. The statement that there is considerable controversy concerning the outcome of treatment for these tumors is correct. Whether anything should be done for most patients with these tumors as long as they remain asymptomatic is still debated.

In our extensive experience with these tumors, my colleagues and I at Johns Hopkins developed a paradigm for management that we think is effective. We do not recommend treatment for the typical optic sheath tumor (usually a meningioma) located only within the optic canal until progressive visual loss is obvious. We then use focused or fractionated radiation. If visual loss continues, decompression of the optic canal and opening the optic sheath may prolong useful vision. If visual loss is rapid, decompression may be performed first.

When hyperostosis involves the orbital walls and sphenoid bone and is associated with proptosis, visual loss, or both, we recommend surgery once symptoms are serious enough to warrant treatment. In this case, the sphenoid bone and anterior clinoid are drilled away extradurally and the optic nerve is decompressed intradurally without attempting to remove the tumor radically. The procedure usually relieves protrusion of the eye and alleviates visual symptoms even when the tumor cannot be removed completely.

Seventy years ago Dandy showed us that small extracanalicular tumors can induce considerable hyperostosis. Treating the hyperostosis by removing the intradural tumor can provide years of cure or palliation. However, if tumor is located internal to the optic sheath, we do not attempt to remove it radically. If radical removal is unfeasible, we believe that the available evidence favors postoperative radiation for these tumors. A minority of 
our group still believes that radiation should follow objective signs of postoperative tumor growth and not be routine.

The authors' review of these unusual tumors and their treatment reminds us of the unanswered questions that attend the management of these tumors.

$$
\text { Donlin M. Long, M.D., Ph.D. }{ }^{1}
$$

